\title{
The topical treatment of old world cutaneous leishmaniasis with gentian violet along with cryotherapy: a pilot single-blind randomized controlled clinical trial
}

\author{
Mozhdeh Sepaskhah ${ }^{[1]}$, Kasra Behdad ${ }^{[1]}$ and Zahra Bagheri ${ }^{[2]}$
}

[1]. Molecular Dermatology Research Center, Shiraz University of Medical Sciences, Shiraz, Iran.

[2]. Department of Biostatistics, Shiraz University of Medical Sciences, Shiraz, Iran.

\begin{abstract}
Introduction: The promising non-clinical antileishmanial effects of gentian violet (GV) encouraged us to evaluate the additive effect of GV on cryotherapy. Methods: For 8 weeks, 59/161 cutaneous leishmaniasis patients/lesions underwent cryotherapy alone (group 1) or cryotherapy accompanied by $1 \% \mathrm{GV}$ application (group 2). The primary endpoint was clinical response. Results: Ultimately, $54.7 \%$ and $45.3 \%$ of the significantly cured lesions belonged to groups 1 and 2, respectively, which was not statistically significant. The clinical response was significantly different between the two groups at the end of the fourth week. Conclusions: Although the clinical response of the two groups was significantly different at the end of the fourth week, application of GV did not increase the efficacy of cryotherapy.
\end{abstract}

Keywords: Leishmaniasis. Treatment. Topical. Gentian violet. Cryotherapy.

Leishmaniasis is a neglected tropical disease caused by the vector-borne protozoan parasite Leishmania. Cutaneous leishmaniasis (CL) is one of the three main clinical presentations of Leishmania infection ${ }^{1}$.

Several treatment modalities have been applied for management of old-world CL with variable efficacies ${ }^{2}$. Although pentavalent antimonies are generally considered the mainstay of CL treatment, the safety of treatment has been challenged ${ }^{3}$.

Some guidelines recommend local therapy for the treatment of limited-size CL lesions ${ }^{4}$.

Several topical therapeutic options have been studied for the treatment of CL. Among them, topical paromomycin with or without combinations, photodynamic therapy, carbon dioxide laser, and thermotherapy resulted in high cure rate, while cryotherapy showed moderate cure rate in a systematic review ${ }^{5}$.

Gentian violet (GV) is a triphenylmethane (TPM) dye discovered in 1861 and has been used as an antibacterial agent

Corresponding author: Dr. Mozhdeh Sepaskhah.

e-mail: sepaskhah_m@yahoo.com

Orcid: 0000-0002-8773-0019

Received 29 June 2018

Accepted 29 November 2018 since $19^{\text {th }}$ century. In addition to its antibacterial activity, GV has antimycotic, antiviral, antihelminthic, and antitrypanosomal effects $^{6}$. Although the results of the study that evaluated in vitro and in vivo antileishmanial activity of GV and 10 other TPMs were promising ${ }^{7}$, to the best of our knowledge, no clinical trial has been conducted to assess the efficacy of GV.

Thus, this pilot single-blind randomized controlled clinical trial was designed to appraise the antileishmanial effect of GV in humans.

Study design and site: This study was a pilot parallel investigator-blind 1:1 randomized controlled clinical trial and was conducted in a teaching hospital at the Shiraz University of Medical Sciences. The study protocol was registered in the Iranian registry of clinical trials (IRCT2017071316557N2).

Patients with clinical diagnosis of leishmaniasis confirmed by direct smear and/or polymerase chain reaction were included in the study. However, if the lesions were absolutely typical for leishmaniasis, the patient was included without laboratory work up. The exclusion criteria were: patients with lesions lasting more than 4 months, receiving systemic or topical antileishmanial treatment or cryotherapy in the recent one month before study, pregnancy, lactation, patients with more than 10 lesions, and lesions located in cartilaginous sites (auricle and nose). 
Patients with clinically infected lesions were recruited after a complete course of oral antibiotic therapy.

Sample size: The sample size calculation was based on the confidence interval approach of Cocks and Torgerson ${ }^{8}$ for pilot randomized trials. Considering the proportion of patients with significant cure approximately equal to $30 \%$ among those treated with cryotherapy combined with gentian violet, a power of $80 \%$ and a significance level of $5 \%$, we would require a pilot sample of 60 participants ( 30 in each group) in order to detect a minimum difference of $10 \%$ between the treatment groups.

Randomization: The patients were randomly allocated to two groups [MEDCALC software version 8 (Ostend, Belgium)] by permuted block randomization (in blocks of size 4). Thirty-three (33) patients were allocated to each group, but some patients did not complete the treatment after allocation. Thus, during followup, there were 30 patients in GV and cryotherapy combination group, whereas 29 patients completed the study in cryotherapy group. The details are shown in Figure 1.

Interventions: Patients in both groups underwent weekly liquid nitrogen cryotherapy using cryospray (Sarmadarman, Tehran, Iran) for 8 weeks. Liquid nitrogen was sprayed approximately $10 \mathrm{~cm}$ away from the lesion for $15 \mathrm{~s}$ with a double freeze-thaw cycle. In addition to cryotherapy, patients in one of the groups applied $1 \%$ gentian violet (GV) ointment twice daily over the lesions for 8 weeks at their home without supervision. The ointment was prepared by dissolving $1 \mathrm{~g}$ of $\mathrm{GV}$ (Merck, Darmstadt, Germany) in $100 \mathrm{~g}$ of Eucerin (Abidaryaco, Isfahan, Iran). The patients were assessed at the beginning of

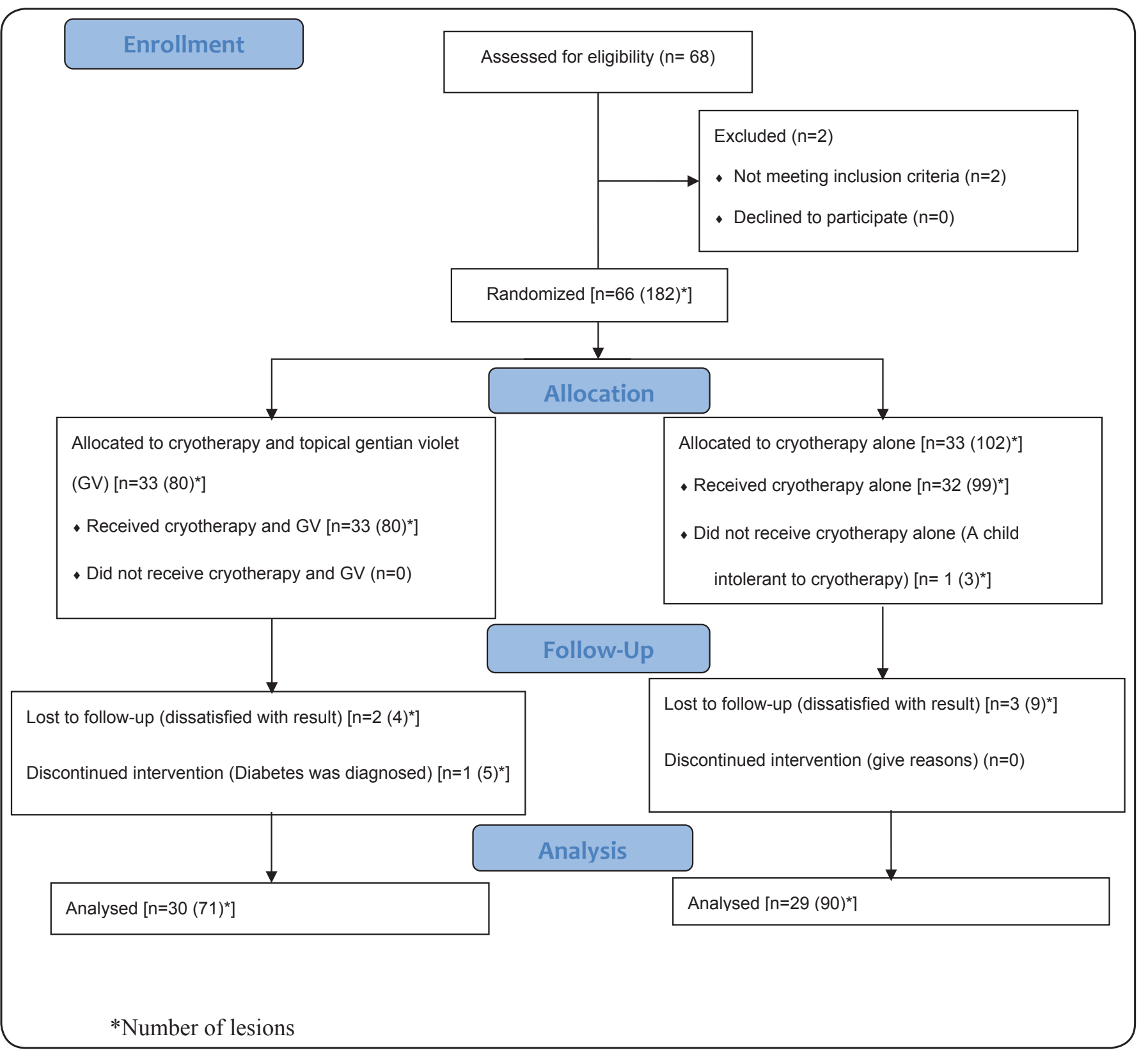

FIGURE 1: CONSORT flow diagram of participants through enrollment, allocation, and follow-up stages of the study 
allocation, and also at the end of $4^{\text {th }}$ and $8^{\text {th }}$ weeks of treatment by an investigator who was unaware of the treatment. The patients in the GV group were recommended to wash the lesion(s) with water and soap or cleanse with alcohol to wash out the purple color of $\mathrm{GV}$ before the $4^{\text {th }}$ and $8^{\text {th }}$ week visits.

Outcome measures: The primary endpoint of this study was defined as clinical cure ${ }^{9}$ as shown below.

Significant cure: more than $75 \%$ reduction in the size of lesion (largest indurated diameter multiplied by the shortest indurated diameter of the lesion, measured by a ruler).

Partial cure: marked by 50-75\% reduction in lesion size.

Failure to respond: less than $50 \%$ reduction in the size of lesion or increase in lesion size.

The clinical cure was reported at the end of the $4^{\text {th }}$ and $8^{\text {th }}$ weeks of treatment.

Ethical considerations: The protocol was approved by the ethical committee of the Shiraz University of Medical Sciences (Ethical code: IR.SUMS.med.REC.1394.29). The ethical principles of the 1975 Declaration of Helsinki were followed. The patients (and parents or legal guardian for patients younger than 18 years) were informed about the study and asked to complete the written consent form.

Data analysis: The data were analyzed using SPSS software version 18 (Chicago, IL, United States). Data of the groups were compared using Chi-square test. The significance level was set at 0.05 .

This study lasted from October 2015 to February 2016 and a total of 68 cases were screened. Sixty-six patients with 182 lesions were recruited into the study. After allocation and during follow- up, the cases declined to 59 with 161 lesions (Figure 1).

The baseline characteristics of the patients and lesions recruited into the study groups are shown in Table $\mathbf{1 .}$

Table 2 presents a comparison of the treatment with cryotherapy combined with GV and the treatment with cryotherapy alone.

At the end of the study, the rate of significant clinical cure was not different between the two groups $(P=0.549)$.

In the $4^{\text {th }}$ week follow-up, $14(70.0 \%)$ and $6(30.0 \%)$ partially cured lesions were treated with cryotherapy combined with $\mathrm{GV}$ and cryotherapy alone, respectively. In the same time, $18(33.3 \%)$ of $\mathrm{GV}$-administered group and $34(66.7 \%)$ of the patients treated with cryotherapy alone healed significantly. The therapeutic responses of the two groups were significantly different in the $4^{\text {th }}$ week follow-up $(P=0.02)$.

No side effect was reported in either group except the transient purple staining of the skin in GV-treated patients.

Despite the variable clinical responses in the $4^{\text {th }}$ week of follow-up, adding gentian violet did not increase the efficacy of cryotherapy in the treatment of cutaneous leishmaniasis.

Investigating topical regimens is an expanding field in pharmacological studies due to their convenience and fewer side effects. Various topical medications have been studied for CL treatment. Few of these topical treatments could be strongly recommended based on qualified studies ${ }^{2}$.

Gentian violet, also known as crystal violet, is a triphenylmethane dye used for the Gram staining of bacteria.

TABLE 1: Baseline characteristics of patients and lesions in cryotherapy combined with gentian violet (GV) and cryotherapy alone groups

\begin{tabular}{|c|c|c|}
\hline Number of patients & 30 & 29 \\
\hline \multicolumn{3}{|l|}{ Methods of diagnosis } \\
\hline Direct smear & 24 & 25 \\
\hline Clinical & 5 & 4 \\
\hline Mean age \pm SD $^{* *}$ (years) & $29.4 \pm 2.8$ & $27.3 \pm 3.2$ \\
\hline \multicolumn{3}{|l|}{ Sex } \\
\hline Male & $18(60 \%)$ & $14(48 \%)$ \\
\hline Upper extremities & $18(25 \%)$ & $45(50 \%)$ \\
\hline Trunk & $11(15 \%)$ & $3(3 \%)$ \\
\hline Head and neck & $0(0 \%)$ & $3(3 \%)$ \\
\hline Mean duration of disease \pm SD (months) & $2.08 \pm 0.16$ & $2.10 \pm 0.19$ \\
\hline
\end{tabular}

*Polymerase chain reaction; **Standard deviation. 
TABLE 2: Comparing effects of cryotherapy combined with gentian violet (GV) and cryotherapy alone on clinical cure of cutaneous leishmaniasis lesions

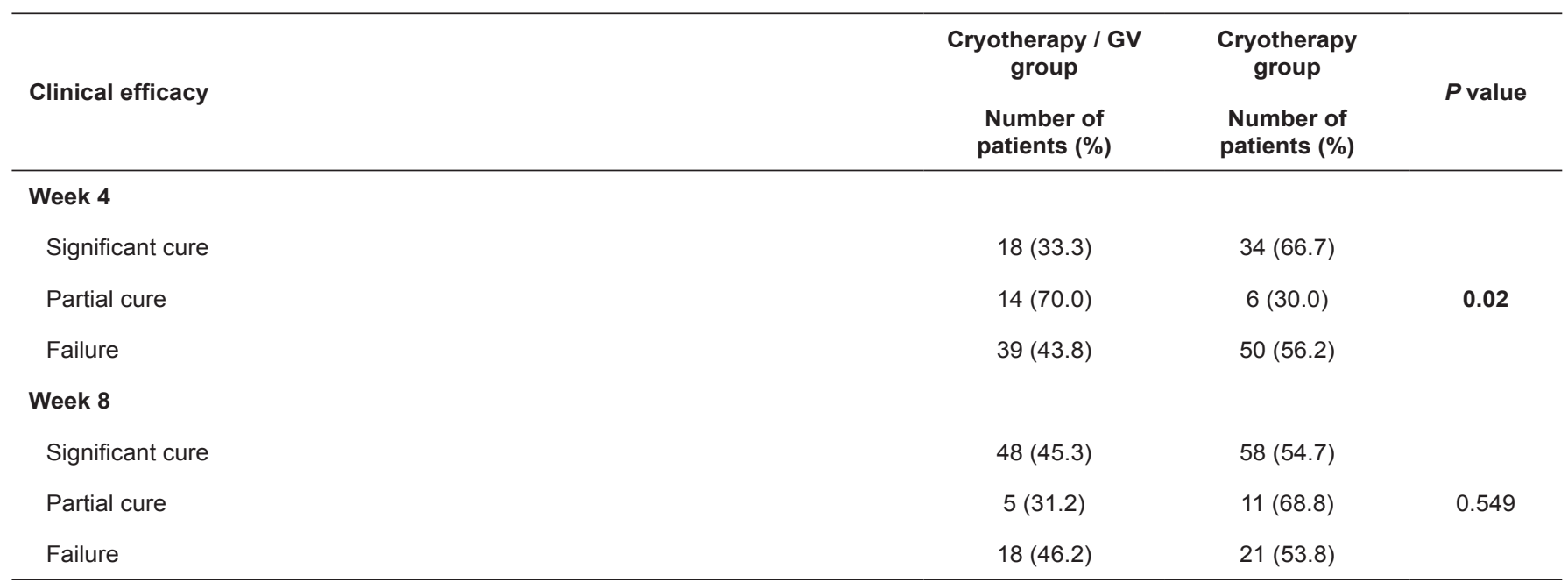

It has also been used clinically for treatment of various infections caused by various Gram-positive and Gram-negative bacteria including methicillin-resistant Staphylococcus aureus, fungi such as Candida, parasitic protozoa such as Trypanosoma Cruzi, parasitic roundworms such as Strongyloides and Enterobius. In addition, antiangiogenic and antitumor activity of GV has also been mentioned ${ }^{6}$.

de Souza Pietra et al. ${ }^{7}$ tested 9 synthetic triphenylmethane derivatives along with GV on Leishmania (L.) amazonensis, Leishmania (V.) braziliensis, and Leishmania major in vitro. $\mathrm{GV}$ was the most effective agent in this study. In BALB/c mice infected with Leishmania (L.) amazonensis and subsequently treated with $1 \% \mathrm{GV}$ gel twice daily, no parasite was detected after 20 days of treatment ${ }^{7}$.

However, these promising results were not reproduced in our clinical trial, which may, at least in part, be explained by the difference in the preparation of the GV (ointment versus gel). To the best of our knowledge, our study is the first clinical trial to evaluate the clinical efficacy of topical GV in the treatment of cutaneous leishmaniasis.

The mechanism of action of GV is not clear exactly. Different hypotheses have been proposed to explain the effects of GV, especially the antimicrobial effects ${ }^{10}$. Among these, two hypotheses are mostly emphasized: 1) inhibition of nicotinamide adenine dinucleotide phosphate (NADPH) oxidase and 2) formation of a covalent complex between $\mathrm{GV}$ and thioredoxin reductase 2 (TrxR2) in mitochondria. The latter mechanism was considered to be more admissible explanation for the role of GV in the treatment of leishmaniasis ${ }^{6}$. TrxR2 is also considered as the target for GV in treatment of cancer and another parasitic infection, malaria ${ }^{11}$.

Although gastrointestinal and hematological side effects as well as carcinogenicity have been reported in rodents following the systemic use of GV, there is no evidence of significant systemic toxicity following external topical application of $\mathrm{GV}^{12}$.

Limitations on the parameters essential for ideal efficacy of topical formulations may explain the discrepancy between the outcomes of in vitro and animal model studies on antileishmanial effect of GV and clinical outcome in our study. Designing more efficient formulations by emerging delivery systems like liposomes, microsponges, lipid nanoparticles, polymeric particles, dendrimers, dendritic-core multishell nanotransporters or even appropriately designing conventional formulations may improve clinical efficacy of topical GV in treating $\mathrm{CL}^{13,14}$.

Besides this limitation in topical medication formulation, our study results may be limited by the small number of patients and lack of follow-up after cessation of treatment. Additionally, we did not determine the parasite species in our study; however, the most common species in our province causing leishmaniasis is Leishmania major ${ }^{15}$.

In conclusion, despite the variable therapeutic effects of GVadded cryotherapy and cryotherapy alone in the early stages of treatment, topical gentian violet ointment did not increase the efficacy of cryotherapy in the treatment of CL.

Acknowledgments: This paper was extracted from the thesis written by K. Behdad M.D. as a part of the requirements for the degree of specialty in dermatology. He was financially supported by a grant (No. 94-01-01-9107) from Research deputy of Shiraz University of Medical Sciences. The authors are grateful to Dr. Sh. Yazdanpanah and Dr. Sh. Habibi for their contribution in patient referring.

Conflict of Interest: The authors declare that there is no conflict of interest.

Financial Support: This work has been financially supported by grant (No. 94-01-01-9107) from Research deputy of Shiraz University of Medical Sciences.

\section{REFERENCES}

1. World Health Organization (WHO). Leishmaniasis. Geneva: WHO; 2017.

2. González U, Pinart M, Reveiz L, Alvar J. Interventions for Old World cutaneous leishmaniasis. Cochrane Database Syst Rev. 2008;(4):CD005067. 
3. Mlika RB, Hamida MB, Hammami H, Jannet SB, Badri T, Fenniche $\mathrm{S}$, et al. Should we continue to indicate meglumine antimoniate as first-line treatment for cutaneous leishmaniasis in Tunisia. Dermatol Ther. 2012;25(6):615-8.

4. Copeland NK, Aronson NE. Leishmaniasis: treatment updates and clinical practice guidelines review. Curr Opin Infect Dis. 2015;28(5):426-37.

5. Wolf Nassif P, DE Mello TFP, Navasconi TR, Mota CA, Demarchi IG, Aristides SMA, et al. Safety and efficacy of current alternatives in the topical treatment of cutaneous leishmaniasis: a systematic review. Parasitology. 2017;144(8):995-1004.

6. Maley AM, Arbiser JL. Gentian violet: a $19 \mathrm{t}^{\mathrm{h}}$ century drug reemerges in the $21^{\text {st }}$ century. Exp Dermatol. 2013;22(12):775-80.

7. de Souza Pietra RCC, Rodrigues LF, Teixeira E, Fried L, Lefkove B, Rabello A, et al. Triphenylmethane derivatives have high in vitro and in vivo activity against the main causative agents of cutaneous leishmaniasis. PLoS One. 2013;8(1):e51864.

8. Cocks K, Torgerson DJ. Sample size calculations for pilot randomized trials: a confidence interval approach. J Clin Epidemiol. 2013;66(2):197-201.

9. Khatami A, Talaee R, Rahshenas M, Khamesipour A, Mehryan P, Tehrani S, et al. Dressings combined with injection of meglumine antimoniate in the treatment of cutaneous leishmaniasis: a randomized controlled clinical trial. PLoS One. 2013;8(6):e66123.

10. Docampo R, Moreno SN. The metabolism and mode of action of gentian violet. Drug Metab Rev. 1990;22(2-3):161-78.

11. Zhang X, Zheng Y, Fried LE, Du Y, Montano SJ, Sohn A, et al. Disruption of the mitochondrial thioredoxin system as a cell death mechanism of cationic triphenylmethanes. Free Radic Biol Med. 2011;50(7):811-20.

12. Arsalan Ali S, Ahmed G, Hamiz-ul-Fawwad S, Waqar SA, Saleem A. Gentian violet - A blessing in disguise for the developing world. Burns. 2013;39(6):1326-7.

13. Espuelas S. Conventional formulations and emerging delivery systems for the topical treatment of cutaneous leishmaniasis. Ther Deliv. 2015;6(2):101-3.

14. Carneiro G, Aguiar MG, Fernandes AP, Ferreira LA. Drug delivery systems for the topical treatment of cutaneous leishmaniasis. Expert Opin Drug Deliv. 2012;9(9):1083-97.

15. Akhoundi M, Hajjaran H, Baghaei A, Mohebali M. Geographical distribution of Leishmania species of human cutaneous leishmaniasis in Fars Province, Southern Iran. Iranian J Parasitol. 2013;8(1):85-91. 